\title{
Inhibition of Immune Complex Complement Activation and Neutrophil Extracellular Trap Formation by Peptide Inhibitor of Complement C1
}

\author{
Pamela S. Hair', Adrianne I. Enos ${ }^{2}$, Neel K. Krishna ${ }^{1,3}$ and Kenji M. Cunnion ${ }^{1,2,3,4 *}$ \\ 'Department of Pediatrics, Eastern Virginia Medical School, Norfolk, VA, United States, ${ }^{2}$ Children's Specialty Group, Norfolk, \\ VA, United States, ${ }^{3}$ Department of Microbiology and Molecular Cell Biology, Eastern Virginia Medical School, Norfolk, VA, \\ United States, ${ }^{4}$ Children's Hospital of The King's Daughters, Norfolk, VA, United States
}

OPEN ACCESS

Edited by:

Fabrice Cognasse,

Groupe Sur L'immunité Des

Muqueuses Et Agents Pathogènes

(GIMAP), France

Reviewed by:

Marko Radic,

University of Tennessee College of

Medicine, United States

Peter A. Ward,

University of Michigan,

United States

*Correspondence:

Kenji M. Cunnion

cunniokm@evms.edu

Specialty section:

This article was submitted to Inflammation,

a section of the journa

Frontiers in Immunology

Received: 12 January 2018

Accepted: 05 March 2018

Published: 26 March 2018

Citation:

Hair PS, Enos Al, Krishna NK and

Cunnion KM (2018) Inhibition of

Immune Complex Complement

Activation and Neutrophil Extracellular

Trap Formation by Peptide

Inhibitor of Complement C1.

Front. Immunol. 9:558.

doi: 10.3389/fimmu.2018.00558
Two major aspects of systemic lupus erythematosus (SLE) pathogenesis that have yet to be targeted therapeutically are immune complex-initiated complement activation and neutrophil extracellular trap (NET) formation by neutrophils. Here, we report in vitro testing of peptide inhibitor of complement $\mathrm{C} 1$ (PIC1) in assays of immune complexmediated complement activation in human sera and assays for NET formation by human neutrophils. The lead PIC1 derivative, PA-dPEG24, was able to dose-dependently inhibit complement activation initiated by multiple types of immune complexes (IC), including $\mathrm{C} 1$-anti-C1q IC, limiting the generation of pro-inflammatory complement effectors, including C5a and membrane attack complex (sC5b-9). In several instances, PA-dPEG24 achieved complete inhibition with complement effector levels equivalent to background. PA-dPEG24 was also able to dose-dependently inhibit NET formation by human neutrophils stimulated by PMA, MPO, or immune complex activated human sera. In several instances PA-dPEG24 achieved complete inhibition with NETosis with quantitation equivalent to background levels. These results suggest that PA-dPEG24 inhibition of NETs occurs by blocking the MPO pathway of NET formation. Together these results demonstrate that PA-dPEG24 can inhibit immune complex activation of the complement system and NET formation. This provides proof of concept that peptides can potentially be developed to inhibit these two important contributors to rheumatologic pathology that are currently untargeted by available therapies.

Keywords: immune complex, complement system, neutrophil extracellular traps, NETosis, myeloperoxidase, peptide inhibitor of complement $\mathrm{C} 1$

\section{INTRODUCTION}

The pathogenesis of systemic lupus erythematosus (SLE) is very complex, but two major contributors are immune complex-initiated complement activation and neutrophil extracellular trap (NET) formation. Immune complexes (IC) initiating classical pathway complement activation leading to consumption of C4 and C3 have long been appreciated and clearly contribute to lupus nephritis (1-3).

Abbreviations: NET, neutrophil extracellular trap; MPO, myeloperoxidase; PIC1, peptide inhibitor of complement C1; PA-dPEG24, IALILEPICCQERAA-dPEG24; SLE, systemic lupus erythematosus. 
NETs, however, are more recently recognized as contributing to SLE pathogenesis, with convincing evidence accruing rapidly in recent years (4-6).

The role of anti-C1q antibodies in the blood of SLE patients is an active area of investigation with considerable data accumulating to demonstrate a strong association between the presence of anti-C1q antibodies and lupus nephritis $(7,8)$. Investigators have also shown that anti-C1q antibodies from SLE patients bound to a surface in an ELISA-type assay can activate the classical and lectin pathways (9). Thus, anti-C1q antibodies appear to be important in pathogenesis and deserve consideration when modeling SLElike IC.

It is known that IC can activate complement generating complement effectors (e.g., C5a, sublytic concentrations of membrane attack complex, etc.) that interact with and can stimulate human neutrophils (10-13). However, articles describing that IC can induce neutrophils to generate NETs have focused on the role of Fc receptors in this process (14-17). These data demonstrate a link between IC and NETs, but to our knowledge the contribution of complement activation in this process remains unexplored. The reverse scenario, NETosing neutrophils activating complement, has previously been reported by Yuen et al (18).

An article by Akong-Moore et al. (19) suggested that a major pathway of NET formation can be mediated by myeloperoxidase (MPO) via its primary function of generating hypochlorous acid from hydrogen peroxide $\left(\mathrm{H}_{2} \mathrm{O}_{2}\right)$ and chloride ion. Another article by Kirchner et al. showed that NADPH oxidase and MPOderived reactive oxygen species are critical for the formation of NETs (20). Studies reported by Parker et al. demonstrated that PMA-stimulated NET formation could be inhibited with ABAH, an MPO inhibitor $(21,22)$. These findings suggest that it might be possible to block NET formation by utilizing other MPO inhibitors.

Peptide inhibitor of complement C1 (PIC1) is a family of more than 70 peptides of related sequences that inhibit the classical pathway of complement by binding and blocking activation of the initiating component of the cascade, $\mathrm{C} 1(23,24)$. The original viral-derived peptides have undergone substantial rational drug design to optimize classical pathway complement inhibition and increase solubility, yielding PA-dPEG24. The lead compound, PA-dPEG24, is a 15 amino acid PEGylated molecule that has been shown to inhibit antibody-initiated complement-mediated hemolysis in vitro and in vivo $(25,26)$. We have recently shown that PA-dPEG24 can inhibit the peroxidase activity of MPO in sputum from cystic fibrosis patients (27). We have subsequently shown that PA-dPEG24 can inhibit the peroxidase activity of other heme-based molecules via interaction with the heme ring (28). In this manuscript, we explore the extent to which PA-dPEG24 can inhibit immune complex-initiated complement activation as well as inhibit NET formation.

\section{MATERIALS AND METHODS}

\section{Ethics Statement}

Blood from healthy donors was obtained with written informed consent under an Eastern Virginia Medical School IRB approved protocol, 02-06-EX 0216.

\section{Reagents}

PA-dPEG24 (IALILEPICCQERAA-dPEG24) was manufactured by PolyPeptide Group (San Diego, CA, USA) to $\geq 95 \%$ purity verified by HPLC and mass spectrometry analysis. Lyophilized PA-dPEG24 was solubilized in normal saline with $0.01 \mathrm{M}$ $\mathrm{Na}_{2} \mathrm{HPO}_{4}$ buffer to $37.5 \mathrm{mM}$. Purified MPO was purchased from Lee Biosolutions (Maryland Heights, MO, USA). Intravenous immune globulin was purchased from Baxter Healthcare Corporation (Westlake Village, CA, USA), ovalbumin from Sigma Aldrich (St. Louis, MO, USA), and the antiovalbumin antibody from Abcam (Cambridge, MA, USA). Goat anti-C1q and human C1 were purchased from Complement Technology (Tyler TX, USA). PMA (Phorbol 12-myristate 13-acetate) and $\mathrm{H}_{2} \mathrm{O}_{2}$ were purchased from Fisher Scientific (Waltham, MA, USA). Oxidized PA-dPEG24 was prepared by adding $\mathrm{H}_{2} \mathrm{O}_{2}$ at a final concentration of $0.3 \%$ for $10 \mathrm{~min}$ at room temperature. Removal of residual $\mathrm{H}_{2} \mathrm{O}_{2}$ was performed by incubating the mixture at $60^{\circ} \mathrm{C}$ for $1 \mathrm{~h}$.

\section{Buffers}

Complement permissive $\mathrm{GVBS}^{++}$buffer is veronal-buffered saline with $0.1 \%$ gelatin, $0.15 \mathrm{mM} \mathrm{CaCl}_{2}$, and $1 \mathrm{mM} \mathrm{MgCl}_{2}$ (29). Complement inhibitory buffer $\mathrm{GVBS}^{--}$is a veronal-buffered saline with $0.1 \%$ gelatin and $10 \mathrm{mM}$ EDTA.

\section{Pooled Normal Human Sera (NHS)}

Pooled NHS was prepared as previously described (29).

\section{Immune complex activation of NHS}

Normal human sera was stimulated with three different types of IC to induce complement activation as follows. Heat-aggregated IgG (Agg-IgG) was generated by incubating intravenous immune globulin at $50 \mathrm{mg} / \mathrm{ml}$ at $63^{\circ} \mathrm{C}$ for $30 \mathrm{~min}$ (30). Ovalbuminantiovalbumin IC were made by incubating $0.01 \mathrm{ml}$ antiovalbumin antibody with an equal volume of ovalbumin, at $0.25 \mathrm{mg} /$ $\mathrm{ml}$, at $37^{\circ} \mathrm{C}$ for $30 \mathrm{~min}$, and then storing at $4^{\circ} \mathrm{C}$ overnight. C1 IC were formed by incubating $0.02 \mathrm{ml}$ anti-C1q goat sera with $5 \mu \mathrm{l}$ of $\mathrm{C} 1$, at $200 \mu \mathrm{g} / \mathrm{ml}$, at $30^{\circ} \mathrm{C}$ for $30 \mathrm{~min}$, and then placing in an ice water bath. For C5a and C5b-9 assays, activation of NHS was performed by pre-incubating 5\% NHS with titrating concentrations of PA-dPEG24 in $0.3 \mathrm{ml}$ of $\mathrm{GVBS}^{++}$buffer for $30 \mathrm{~min}$ at room temperature. Then $2 \mu \mathrm{l}$ of either heat-aggregated IVIg, or ovalbumin IC, or $5 \mu \mathrm{l}$ of $\mathrm{C} 1$-anti-C1q IC was added to the mix for $30 \mathrm{~min}$ at $37^{\circ} \mathrm{C}$. This reaction was stopped with the addition of an equal volume of $\mathrm{GVBS}^{--}$. For iC3b detection, 1\% NHS was used and the rest of the protocol remained the same.

\section{ELISAs}

Samples were assayed using C5a, iC3b, and SC5b-9 ELISAs. A C5a ELISA kit (R\&D Systems) was used per the manufacturer's instructions. ELISAs for $\mathrm{iC} 3 \mathrm{~b}$ and SC5b-9 were performed as previously described (31). The iC3b ELISA uses a goat anti-human C3 antibody (Complement Technology, Tyler, TX, USA) for capture, and a mouse anti-human iC3b antibody (Quidel, San Diego, CA, USA) for probing, and a goat anti-mouse HRP antibody for detection. The SC5b-9 ELISA uses a rabbit anti-human SC5b-9 antibody (Complement Technology) for capture, a mouse antihuman SC5b-9 monoclonal antibody (Quidel) for probing, and 
a chicken anti-mouse HRP antibody for detection. Colorimetric detection was performed with $\mathrm{TMB}$, stopped with $\mathrm{H}_{2} \mathrm{SO}_{4}$, and read on a BioTek Synergy HT plate reader at $450 \mathrm{~nm}$.

\section{Purified Neutrophils and Neutrophil Lysate}

Neutrophils from the blood of healthy volunteers were purified from heparinized blood by Hypaque-Ficoll step gradient centrifugation, dextran sedimentation, and hypotonic lysis, as previously described (32).

\section{NET Assay in Microtiter Plate}

The formation of NETs was induced by incubating $2.0 \times 10^{5}$ neutrophils per well in a 96-well tissue culture plates with RPMI media alone, or adding $0.05 \%$ of $\mathrm{H}_{2} \mathrm{O}_{2}$, or $12 \mathrm{nM} \mathrm{PMA}$, or $8 \mu \mathrm{g} / \mathrm{ml}$ $\mathrm{MPO}$, or PA-dPEG24 at various concentrations. For immune complex sera-induced NET formation, activated sera were made by adding $5 \mu \mathrm{l}$ of ovalbumin-antiovalbumin immune complex to $5 \%$ NHS in $0.3 \mathrm{ml}$ of $\mathrm{GVBS}^{++}$. This combination was allowed to incubate for $30 \mathrm{~min}$ at $37^{\circ} \mathrm{C}$, and then $0.05 \mathrm{ml}$ was added to the neutrophils in RPMI. Cells were then incubated for $1.5 \mathrm{~h}$ at $37^{\circ} \mathrm{C}$ in $5 \% \mathrm{CO}_{2}$ incubator.

\section{Ellman's Reagent Assay}

Samples were mixed 1:10 into an $80 \mu \mathrm{g} / \mathrm{ml}$ solution of Ellman's Reagent (Thermo Scientific Pierce, Waltham, MA, USA) diluted in reaction buffer $(0.1 \mathrm{M}$ sodium phosphate with $1 \mathrm{mM}$ EDTA, $\mathrm{pH}$ 8.0) and allowed to incubate at room temperature for $15 \mathrm{~min}$. A standard curve was generated using cysteine hydrochloride monohydrate (Sigma Aldrich, St. Louis, MO, USA) at concentrations ranging from 0.25 to $1.5 \mathrm{mM}$ in the reaction buffer mixed with the Ellman's reagent at the same proportion and incubated the same as the samples. Samples and standards were read at an absorbance of $412 \mathrm{~nm}$ in a BioTek Synergy HT plate reader and a linear regression was generated and used to analyze the samples yielding a calculation of $\mathrm{mM}$ sulfhydryl groups relative to a cysteine standard.

\section{Quantitation of NET Formation}

Free DNA was measured by PicoGreen in the supernatant recovered from the NET microtiter plate well assay (19). Five hundred units of monococcal nuclease (Fisher) were added to each well to allow for digestion of released extracellular DNA for $10 \mathrm{~min}$ in $37^{\circ} \mathrm{C}$ incubator. The preparation was then aliquoted into an adjacent well and mixed 1:1 with prepared PICO green reagent (Fisher). The fluorescence was then quantified on a BioTek microplate reader at excitation $485 \mathrm{~nm} /$ emission $528 \mathrm{~nm}$.

\section{NET Formation for Fluorescence Microscopy}

Purified human neutrophils were assayed on a glass slide as follows. Cells were combined with RPMI media and the indicated stimuli as mentioned above in a tube and then aliquoted onto a glass slide circled with a hydrophobic slide marker. The slides were incubated for $1.5 \mathrm{~h}$ at $37^{\circ} \mathrm{C}$ in a $5 \% \mathrm{CO}_{2}$ incubator at $37^{\circ}$ for $1.5 \mathrm{~h}$. Slides were fixed overnight with $4 \%$ paraformaldehyde at $4^{\circ} \mathrm{C}$. For all staining, the following conditions were used.
Slides were washed in PBS and incubated in blocking solution ( $2 \%$ normal goat serum $+2 \%$ bovine serum albumin in PBS) for $1 \mathrm{~h}$ at room temperature. Then the slides were incubated with primary antibody at 1:300 in 2\% BSA in PBS for $1 \mathrm{~h}$ at room temperature. Slides were washed in PBS three times and incubated in fluorescent-labeled secondary antibody at 1:1,000 or DAPI (Southern Biotech) at $0.25 \mathrm{pg} / \mathrm{ml}$ final in $2 \%$ BSA in PBS for $1 \mathrm{~h}$ at room temperature. Slides were then washed three times in PBS and were imaged. Cells were visualized using a DP70 Digital Camera (Olympus Center, Valley Forge, PA, USA), mounted on a BX50, Olympus microscope. Staining antibody pairs used were rabbit anti-MPO (Thermo Scientific) and rabbit anti-histone $\mathrm{H} 3$ (Abcam) with the secondary goat anti-rabbit Alexa Fluor 488 (Novus Biologicals). Also, mouse anti-elastase (Invitrogen) was used with the secondary goat anti-mouse Alexa Fluor 568 (Novus Biologicals).

\section{Statistical Analysis}

Quantitative data were analyzed determining means, SEM, and Student's $t$-test (33) using Excel (Microsoft, Redmond, WA, USA).

\section{RESULTS}

\section{PA-dPEG24 Inhibition of Immune Complex-Initiated Complement Activation}

Our group has previously shown that PA-dPEG24 can inhibit classical pathway-mediated hemolysis in a CH50-type assay (25) as well as inhibit classical pathway-mediated hemolysis in vitro and in vivo in a mismatched transfusion model (26). In order to evaluate the ability of PA-dPEG24 to inhibit immune complexinitiated complement activation, we utilized the archetypal immune complex stimulant of heat-Agg-IgG $(30,34)$ in NHS. We assayed for three important effectors resulting from complement activation, the major proinflammatory anaphylatoxin, C5a, a cleavage product of $\mathrm{C} 3$ activation, $\mathrm{iC} 3 \mathrm{~b}$, and the membrane attack complex, C5b-9 (Figures 1A-C). For each assay, PA-dPEG24 dose-dependently inhibited elaboration of the effector after stimulation with heat-Agg-IgG compared with no inhibitor. Statistically significant inhibition was achieved in each assay at $\geq 0.5 \mathrm{mM}$ PA-dPEG24 ( $p<0.05)$. For C5a, 1 mM PA-dPEG24 lead to a $61 \%$ reduction $(p=0.002)$ compared with heat-Agg-IgG with no inhibitor. These results suggest that PA-dPEG24 can inhibit immune complex-initiated complement activation in human sera.

In order to provide confirmation of the results with heatAgg-IgG, we then tested the antigen-antibody immune complex most often utilized in animal models of complement activation, ovalbumin, and antiovalbumin $(35,36)$. The ovalbuminantiovalbumin IC were used to stimulate complement activation in NHS and the same three effectors, C5a, iC3b, and C5b-9, were measured. PA-dPEG24 dose dependently inhibited generation of each complement effector with statistically significant inhibition achieved at $\geq 0.25 \mathrm{mM}$ PA-dPEG24 ( $p<0.03)$ compared with ovalbumin-antiovalbumin alone (Figures 2A-C). These results provide additional confidence that PA-dPEG24 can inhibit immune complex-initiated complement activation in human sera. 

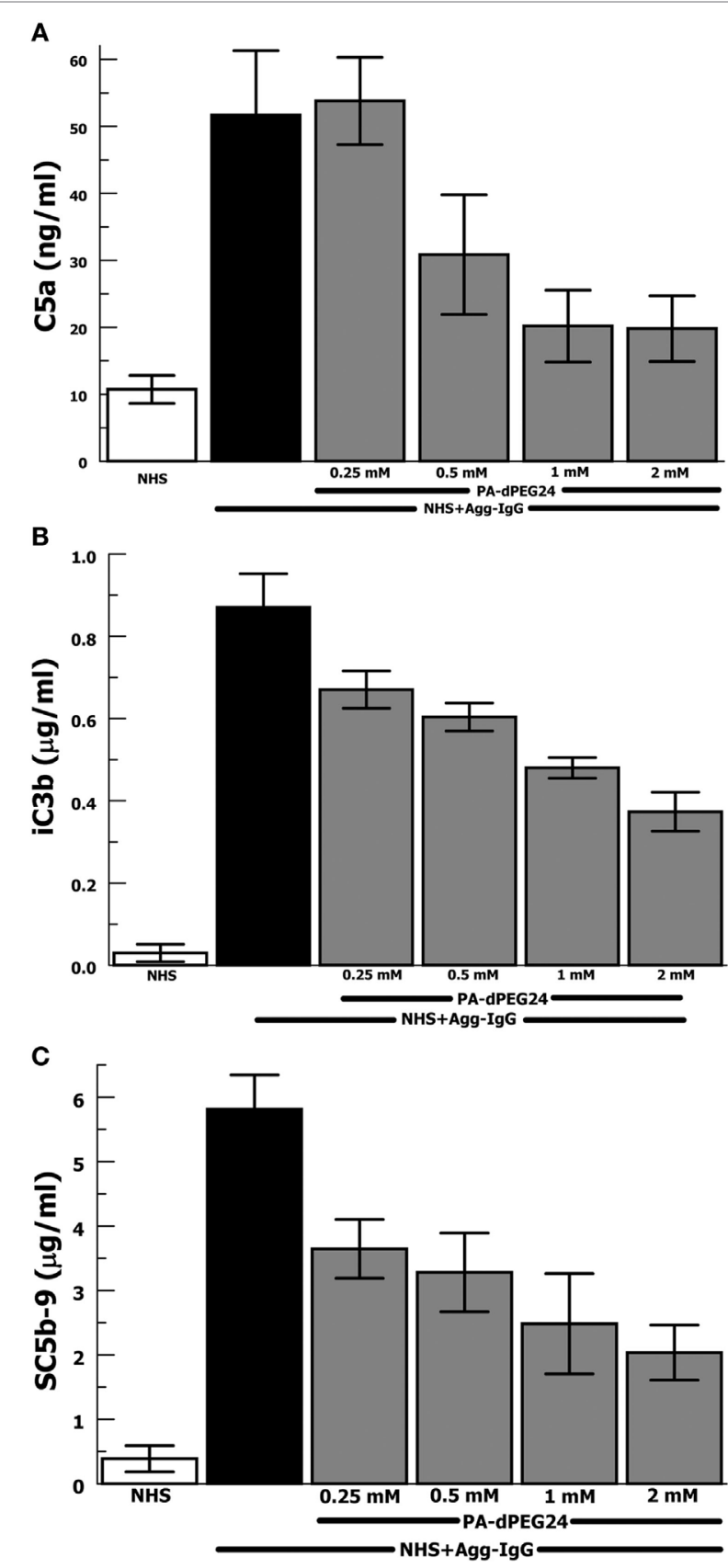

FIGURE 1 | PA-dPEG24 inhibition of heat-aggregated lgG (Agg-lgG) immune complex-initated complement activation assayed by complement effectors.

(A) PA-dPEG24 inhibition of C5a generation in normal human sera (NHS) stimulated with heat-Agg-lgG immune complexes (IC). Data are means of $(n=5)$ independent experiments \pm SEM. (B) PA-dPEG24 inhibition of iC3b generation in NHS stimulated with heat-Agg-IgG IC. Data are means of $(n=4)$ independent experiments \pm SEM. (C) PA-dPEG24 inhibition of SC5b-9 generation in NHS stimulated with heat-Agg-IgG IC. Data are means of $(n=6)$ independent experiments \pm SEM.

Due to the importance of anti-C1q antibodies in a subset of patients with SLE, we generated IC with human C1 and anti-C1q antibodies (goat). These IC activated NHS leading to robust generation of C5a, iC3b, and SC5b-9 (Figures 3A-C). PA-dPEG24

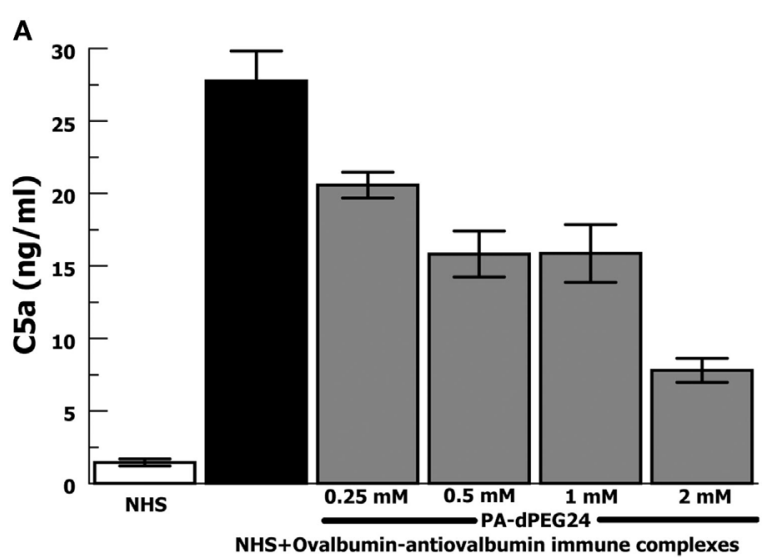

B
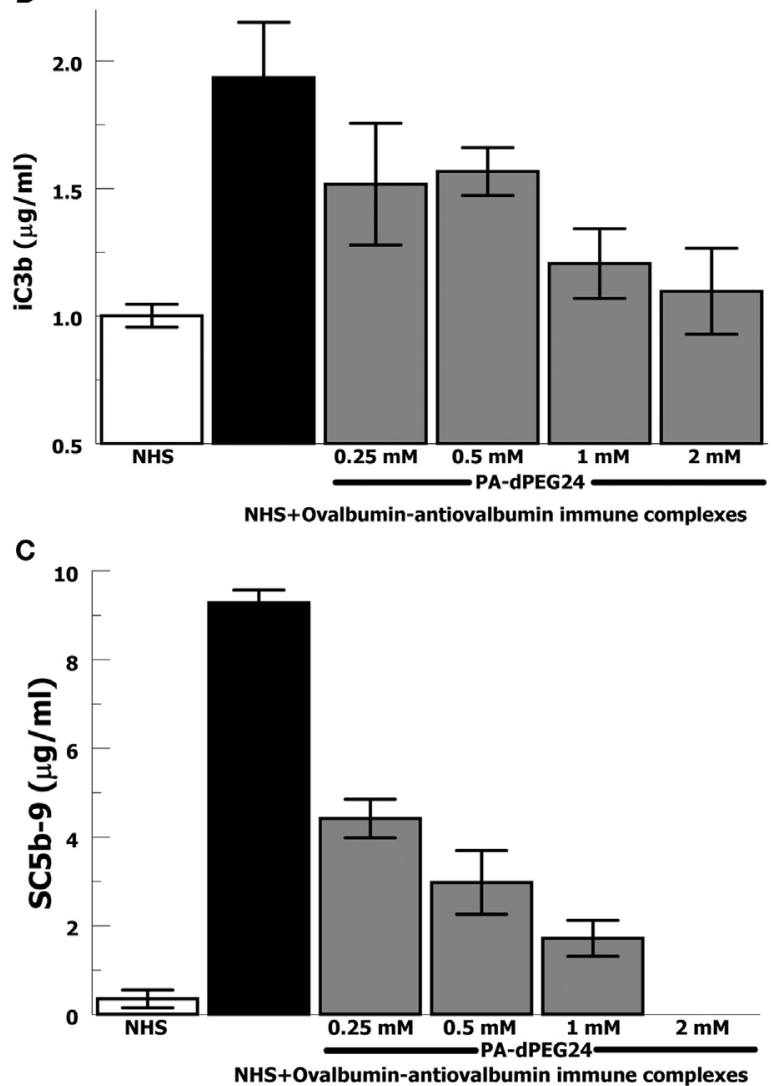

FIGURE 2 | PA-dPEG24 inhibition of ovalbumin-antiovalbumin immune complex-initated complement activation assayed by complement effectors. (A) PA-dPEG24 inhibition of C5a generation in normal human sera (NHS) stimulated with ovalbumin-antiovalbumin immune complexes (IC). Data are means of $(n=4)$ independent experiments \pm SEM. (B) PA-dPEG24 inhibition of iC3b generation in NHS stimulated with ovalbumin-antiovalbumin IC. Data are means of $(n=4)$ independent experiments \pm SEM. (C) PA-dPEG24 inhibition of SC5b-9 generation in NHS stimulated with ovalbuminantiovalbumin IC. At 2 mM PA-dPEG24 the measured SC5b-9 was at the lower limit of detection. Data are means of $(n=3)$ independent experiments \pm SEM

dose-dependently inhibited $\mathrm{C} 1$-anti-C1q generation of $\mathrm{C} 5 \mathrm{a}$ in NHS at each concentration $(p \leq 0.03)$. C1-anti-C1q generation of iC3b was inhibited by 2 mM PA-dPEG24 $(p<0.02)$ to a level 


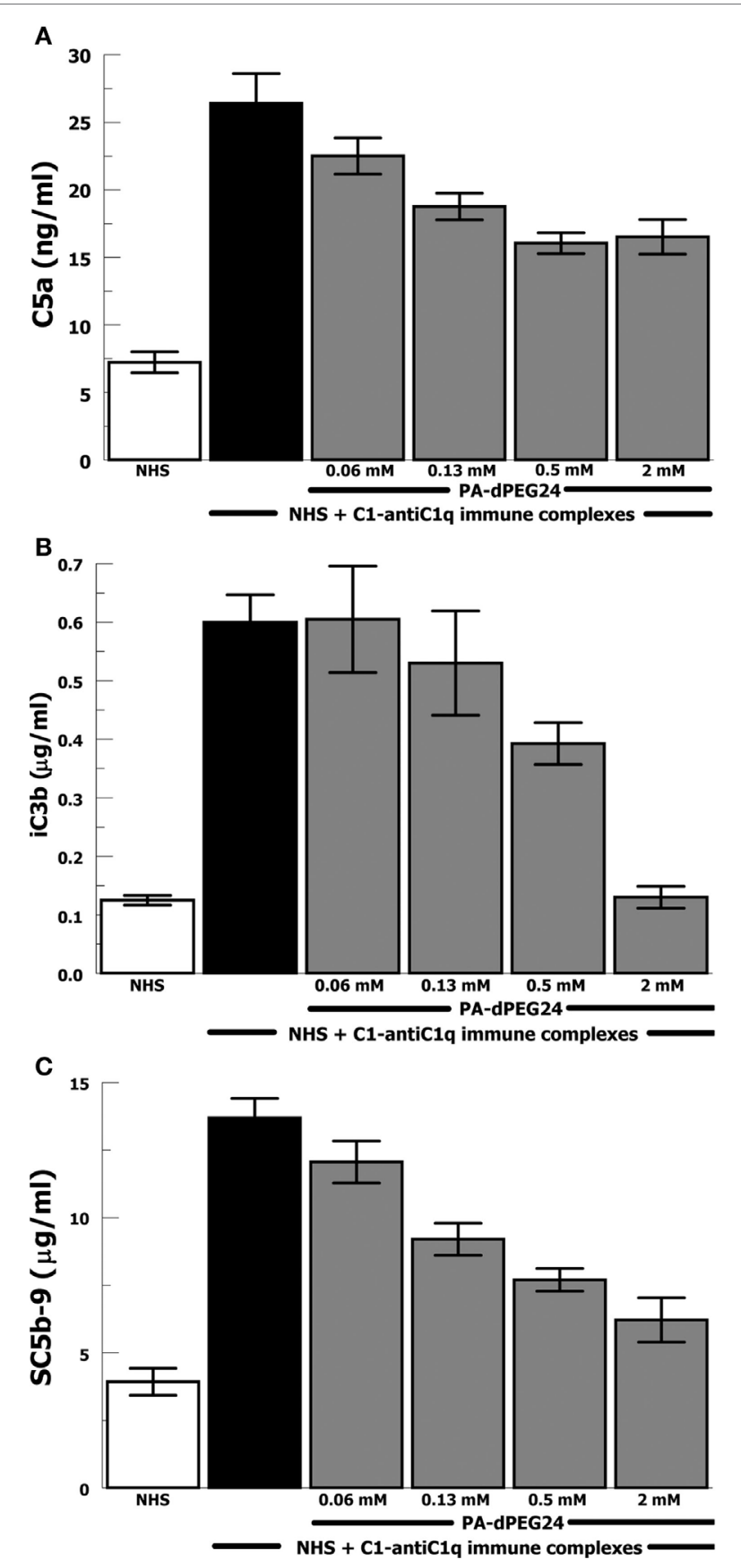

FIGURE 3 | PA-dPEG24 inhibition of C1-anti-C1q immune complex-initated complement activation assayed by complement effectors. (A) PA-dPEG24 inhibition of $\mathrm{C} 5$ a generation in normal human sera (NHS) stimulated with C1-anti-C1q immune complexes (IC). Data are means of $(n=4)$ independent experiments \pm SEM. (B) PA-dPEG24 inhibition of iC3b generation in NHS stimulated with C1-anti-C1q IC. Data are means of $(n=4)$ independent experiments \pm SEM. (C) PA-dPEG24 inhibition of SC5b-9 generation in NHS stimulated with C1-anti-C1q IC. Data are means of $(n=4)$ independent experiments \pm SEM.

similar to NHS baseline. PA-dPEG24 dose-dependently inhibited C1-anti-C1q generation of SC5b-9 for concentrations $\geq 0.13 \mathrm{mM}$ $(p<0.03)$. These results show that PA-dPEG24 can inhibit
C1-anti-C1q immune complex-initiated complement activation in human sera.

\section{PA-dPEG24 Inhibition of PMA-Initiated NET Formation by Human Neutrophils}

In order to evaluate whether PA-dPEG24 can inhibit NET formation, we used purified human neutrophils and the commonly utilized stimulus phorbol 12-mystate 13-acetate (PMA) similar to methods described by Akong-Moore et al. (19). Two major components of NETs are extracellular DNA and myeloperoxidase which were visualized with DNA and anti-MPO antibody, respectively. Human neutrophils stimulated with PMA and $\mathrm{H}_{2} \mathrm{O}_{2}$ generated many NETs visualized by fluorescence microscopy, demonstrating extracellular DNA and extracellular MPO (Figure 4). In the presence of 5 mM PA-dPEG24, PMA and $\mathrm{H}_{2} \mathrm{O}_{2}$ did not generate NETs that could be identified by fluorescence microscopy. We then quantified NET formation by measuring free DNA in a PicoGreenbased assay from supernatants of human neutrophils stimulated in microtiter plate wells. PA-dPEG24 (5 mM) was able to inhibit free DNA elaboration by 2.6 -fold $(p=0.01)$ in the presence of PMA and $\mathrm{H}_{2} \mathrm{O}_{2}$ compared with no inhibitor (Figure 4). This reduction for PA-dPEG24 was to a level similar to baseline without PMA. We also performed fluorescence microscopy utilizing the same experimental conditions, but probing for additional NET constituents extracellular neutrophil elastase and histone H3 (Figure 5A). Evaluating for these NET components also demonstrated that stimulation with PMA and $\mathrm{H}_{2} \mathrm{O}_{2}$ resulted in copious NET formation, which was inhibited in the presence of PA-dPEG24 (5 mM). In order to determine if the sulfhydryl groups in PA-dPEG24 were critical for inhibition of NET formation, we oxidized PA-dPEG24. The oxidized form of PIC1 demonstrated significantly less inhibition of PMA-initiated NET formation compared with unoxidized PA-dPEG24 $(P=0.006)$ and was not statistically different from the no PA-dPEG24 condition $(P=0.18)$ (Figure 5B). Complete oxidation of PA-dPEG24 sulfhydryl groups was verified using Ellman's reagent which reacts with reduced sulfhydryl groups producing a color change that can be detected at $412 \mathrm{~nm}$ (Figure 5C). These results suggest that PA-dPEG24 can inhibit PMA-stimulated NET formation by human neutrophils.

\section{PA-dPEG24 Inhibition of MPO-Initiated NET Formation by Human Neutrophils}

The results reported by Akong-Moore et al. (19) suggest that MPO is the critical mediator in PMA-stimulated NET formation; however, this was never tested using purified MPO. Thus, we repeated the above experiments with purified human neutrophils substituting purified MPO for PMA as the stimulus for NET formation. Neutrophil stimulation with purified MPO and $\mathrm{H}_{2} \mathrm{O}_{2}$ caused extensive NET formation visualized by DAPI staining and anti-MPO staining (Figure 6). NET formation in the presence of MPO and $\mathrm{H}_{2} \mathrm{O}_{2}$ was blocked with PA-dPEG24 (5 mM). Neutrophils incubated with PA-dPEG24 alone appeared normal by fluorescence microscopy. When NET formation was quantified by PicoGreen measurement, $1.1 \mathrm{mM}$ of PA-dPEG24 lead to a $30 \%(p=0.02)$ reduction in free DNA and $4.5 \mathrm{mM}$ of PA-dPEG24 resulted in a 3.7-fold $(p=0.001)$ reduction in free DNA compared 

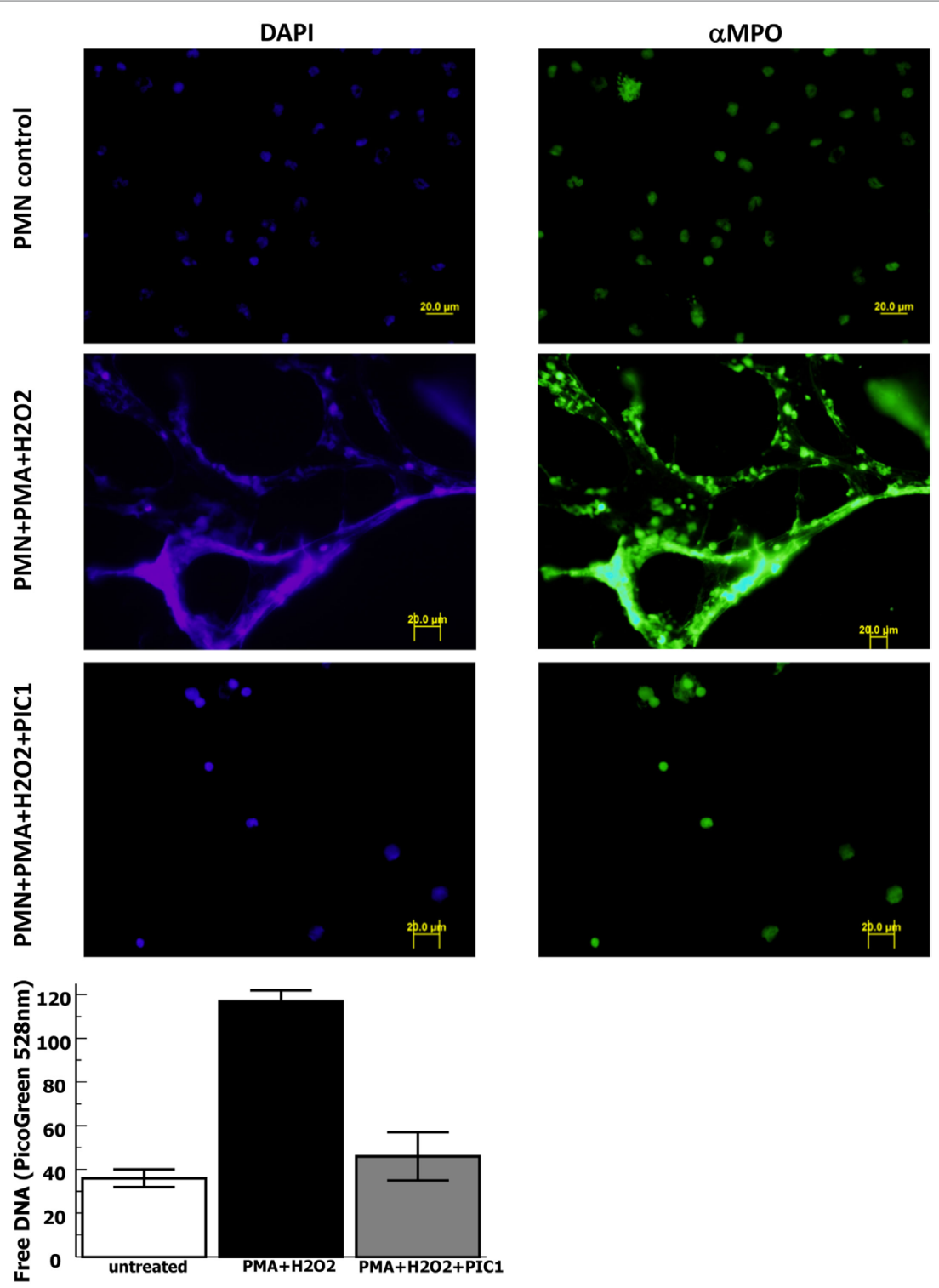

FIGURE 4 | PA-dPEG24 inhibition of PMA-initiated neutrophil extracellular trap (NET) formation with human neutrophils (PMN) assayed by fluorescence microscopy and PicoGreen quantitation of free DNA. The first row shows unstimulated neutrophils, the second row shows neutrophils stimulated with PMA and hydrogen peroxide $\left(\mathrm{H}_{2} \mathrm{O}_{2}\right)$, and third row shows neutrophils stimulated with PMA $+\mathrm{H}_{2} \mathrm{O}_{2}$ in the presence of $5 \mathrm{mM}$ PA-dPEG24 peptide inhibitor of complement C1. The first columns are slides probed with DAPI to visualize DNA and the second column are slides probed with anti-myeloperoxidase antibody. The graph shows PA-dPEG24 (5 mM) inhibition of NET generation by human neutrophils stimulated with PMA $+\mathrm{H}_{2} \mathrm{O}_{2}$ assayed by PicoGreen. Data are means of $(n=3)$ independent experiments $\pm \mathrm{SEM}$.

with stimulation with MPO, but no inhibitor (Figure 6). In the presence of MPO plus 4.5 mM PA-dPEG24, measured free DNA was not statistically different from unstimulated neutrophils. These results suggest that PA-dPEG24 inhibits NET formation via the MPO-mediated pathway.

\section{PA-dPEG24 Inhibition of Immune Complex-Initiated NET Formation by Human Neutrophils}

Next we evaluated for a potential relationship between immune complex-initiated complement-activated human sera and NET formation by human neutrophils, because this has not been previously explored. We activated complement in normal human sera with ovalbumin-antiovalbumin IC as was performed in Figure 2. The immune complex-initiated complement-activated human sera were then incubated with purified human neutrophils resulting in NET formation quantified by free DNA measurement with PicoGreen. We initially evaluated the relative contribution of IC by them compared with immune complex-activated human sera for generation of NETs. The presence of IC by themselves did not significantly $(p=0.39)$ increase NET formation compared with neutrophils alone (Figure 7A). However, immune complex activation of complement in sera increased NET formation 


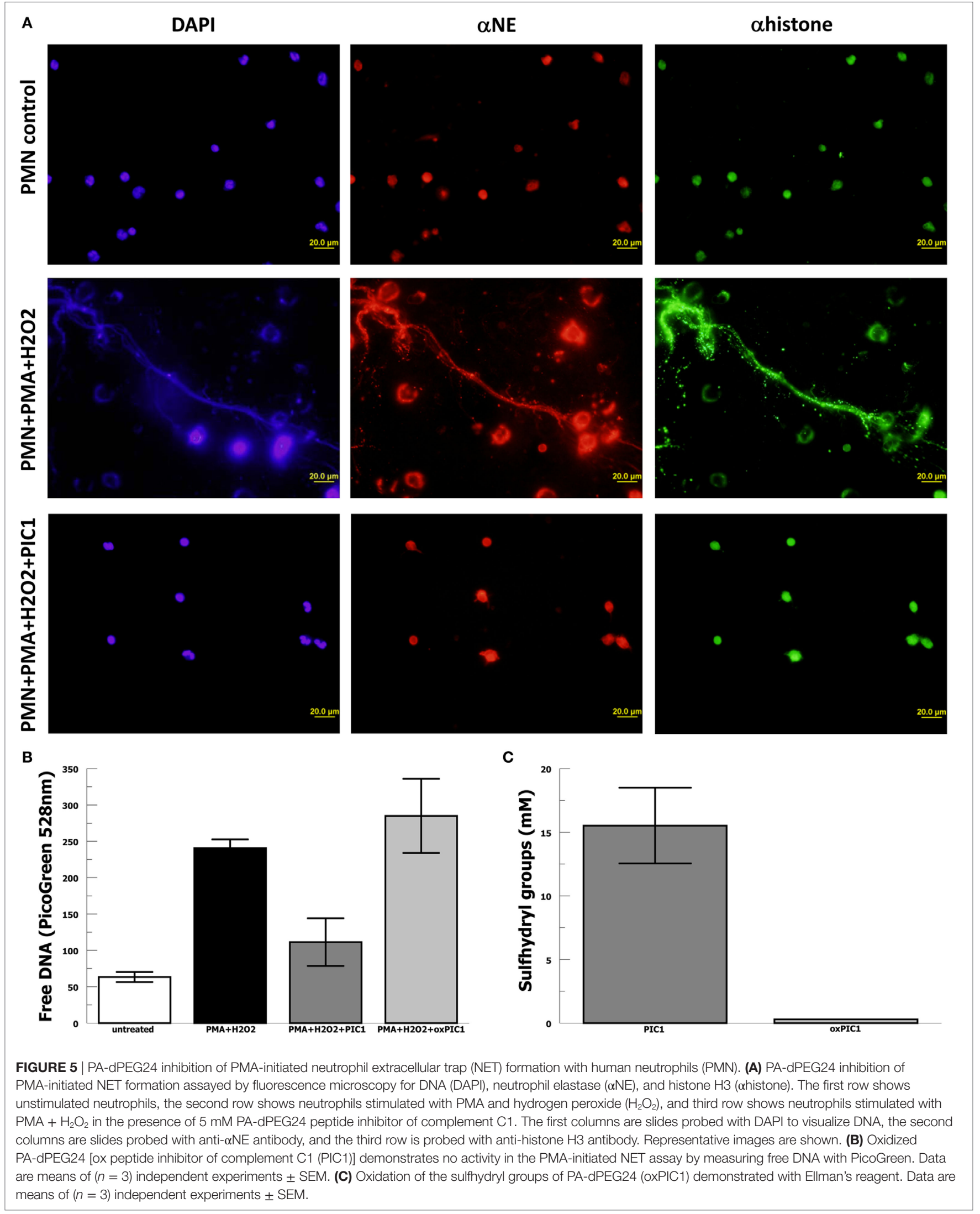




\section{DAPI}
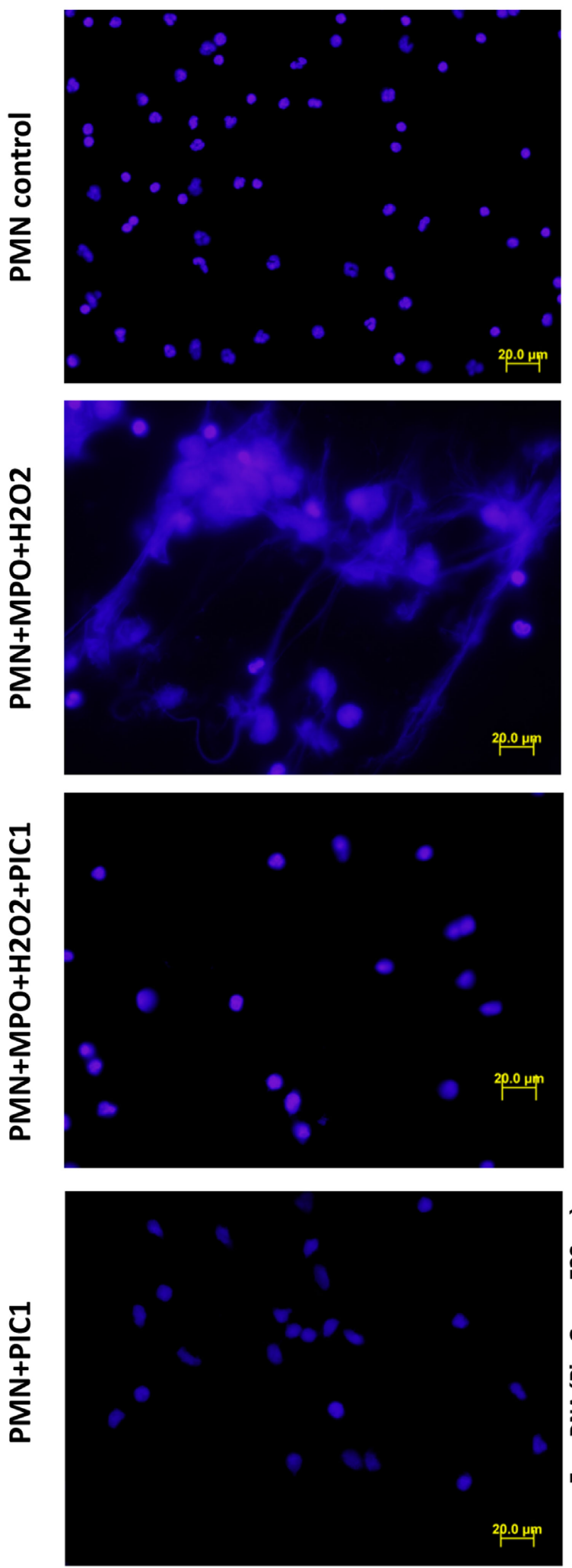

$\alpha M P O$
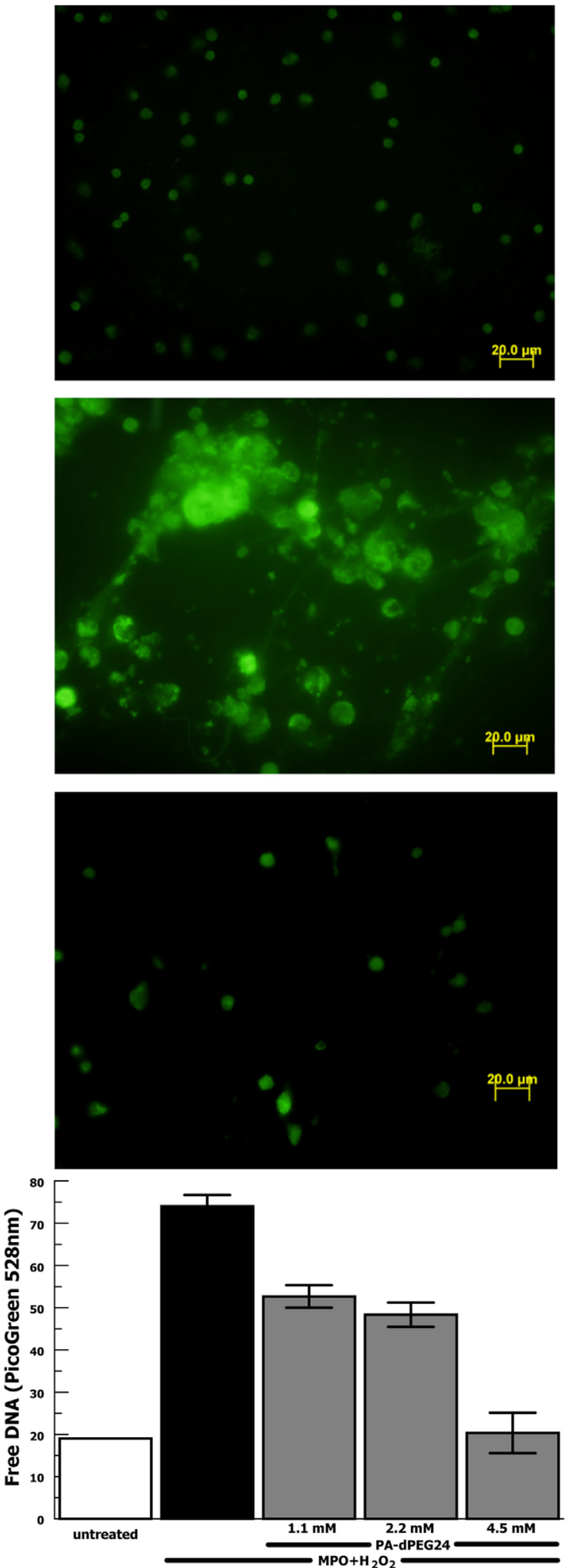

FIGURE 6 | Continued 
FIGURE 6 | PA-dPEG24 inhibition of myeloperoxidase (MPO)-initiated neutrophil extracellular trap (NET) formation with human neutrophils (PMN) assayed by fluorescence microscopy and PicoGreen quantitation of free DNA. The first row shows unstimulated neutrophils, the second row shows neutrophils stimulated with MPO and hydrogen peroxide $\left(\mathrm{H}_{2} \mathrm{O}_{2}\right)$, the third row shows neutrophils stimulated with $\mathrm{MPO}+\mathrm{H}_{2} \mathrm{O}_{2}$ in the presence of PA-dPEG24 [peptide inhibitor of complement C1 (PIC1)], and the fourth column shows neutrophils incubated with PA-dPEG24 (PIC1) only. The first column shows slides probed with DAPI to visualize DNA. The second column shows slides probed with anti-MPO antibody. The graph shows PA-dPEG24 inhibition of NET generation by human neutrophils stimulated with $\mathrm{MPO}+\mathrm{H}_{2} \mathrm{O}_{2}$ assayed by PicoGreen. Data are means of $(n=3)$ independent experiments \pm SEM.

$>20$-fold $(p=0.009)$ compared with IC alone after subtracting the background. These results suggest that immune complexinitiated complement-activated sera are a strong stimulus for NET formation.

We then evaluated whether $\mathrm{H}_{2} \mathrm{O}_{2}$ further enhanced NET formation by immune-complex-activated human sera (ICsera) and found that it approximately doubled the signal (Figure 7B). Testing of PA-dPEG24 inhibition of NETosis was performed with PA-dPEG24 being added after complement activation of the sera by IC that had already been allowed to occur; therefore, any effect of PA-dPEG24 on NETosis happened downstream of complement activation. In the presence immune complex-initiated complement-activated sera and $\mathrm{H}_{2} \mathrm{O}_{2}$, $2.2 \mathrm{mM}$ PA-dPEG24 decreased free DNA by $23 \%(p=0.037)$ and $4.5 \mathrm{mM}$ PA-dPEG24 decreased free DNA by threefold $(p<0.001)$ compared with no inhibitor (Figure 7B). These conditions were also visualized by fluorescence microscopy (Figure 7C). In the presence of PA-dPEG24 (5 mM) and ICsera, no NETs were identified by fluorescence microscopy. These findings demonstrate immune complex-initiated complementactivated human sera can stimulate human neutrophils to form NETs and that this can be inhibited with PA-dPEG24. Taken together, these experiments consistently show that PA-dPEG24 can inhibit NET formation by human neutrophils initiated by various stimulants.

\section{DISCUSSION}

The experiments shown above demonstrate that PA-dPEG24 can inhibit immune complex-initiated complement activation and the generation of pro-inflammatory complement effectors. This suggests that complement inhibitory peptides could potentially moderate aspects of pathogenesis in diseases where immune complex activation of the complement system plays a vital role. Additionally, we utilized $\mathrm{C} 1$ and anti-C1q antibodies as a novel immune complex modeling a type of immune complex that could be predicted to be formed in the plasma of SLE patients with circulating anti-C1q antibodies. PA-dPEG24 also blocked complement activation by $\mathrm{C} 1$-anti-C1q IC, consistent with the other immune complex types tested.

We have previously shown that PA-dPEG24 inhibits hemolysis of antibody-sensitized erythrocytes in vitro and in vivo $(25,26)$. The studies described here expand upon these observations demonstrating that PA-dPEG24 can inhibit immune complex-initiated complement activation. Thus, there is consistency in PA-dPEG24 inhibition of the classical complement pathway for various stimuli.

Our NETosis experiments demonstrate that immune complex-initiated complement-activated human sera can initiate NET formation. This adds to prior observations that IC by themselves can initiate NET formation via Fc receptors (14-17). Under the experimental conditions we used, the contribution of immune complex-initiated complement activation to NET formation was much greater than that of IC alone. Given the presence of IC in several rheumatologic diseases, including active SLE disease, it is reasonable to hypothesize that immune complex-initiated complement activation may contribute to NET formation in SLE and potentially other rheumatologic diseases.

These experiments also show that PA-dPEG24 can inhibit NET formation by human neutrophils initiated by PMA, MPO, or immune complex-activated human sera. Given that PMA has been shown to stimulate NET formation via an MPO-mediated pathway (19) and our results utilizing purified MPO, it is likely that PA-dPEG24 blocks NET formation by inhibiting MPO (27). The ability of PA-dPEG24 to inhibit NET formation after immune complex-initiated complement activation of human sera has occurred, suggests that this mechanism of NET formation may also occur via an MPO-mediated pathway. This adds immune complex-initiated complement activation as a new stimulus for MPO-mediated NET formation to others described previously (19-21).

Together, these data show that the PA-dPEG24 peptide can block both immune complex-initiated complement activation and inhibit NETosis. The vital role of NETs in autoimmune diseases was nicely reviewed by Brinkmann and Zychlinsky (37) describing the role of NETs in SLE noting the potential role of increased C1q deposition inhibiting DNaseI and preventing NET degradation. Given that the peptide core of PA-dPEG24 strongly binds $\mathrm{C1q}$ (25), warrants future studies to evaluate a potential effect on NET degradation. A more recent review of NETosis contribution to autoimmune diseases written by Gupta and Kaplan (38) details the role of NETs in SLE, ANCA-associated vasculitis, rheumatoid arthritis, antiphospholipid antibody syndrome, type 1 diabetes, and renal inflammatory diseases. This provides proof of concept that peptides can potentially be developed to inhibit these two important contributors to rheumatologic pathology that is currently untargeted by available therapies. The next steps for evaluation of PA-dPEG24 will be testing in animal models of immune complex-mediated diseases and animal models of NET-mediated diseases.

\section{DATA AVAILABILTY}

The raw data supporting the conclusions of this manuscript will be made available by the authors, without undue reservation, to any qualified researcher. 

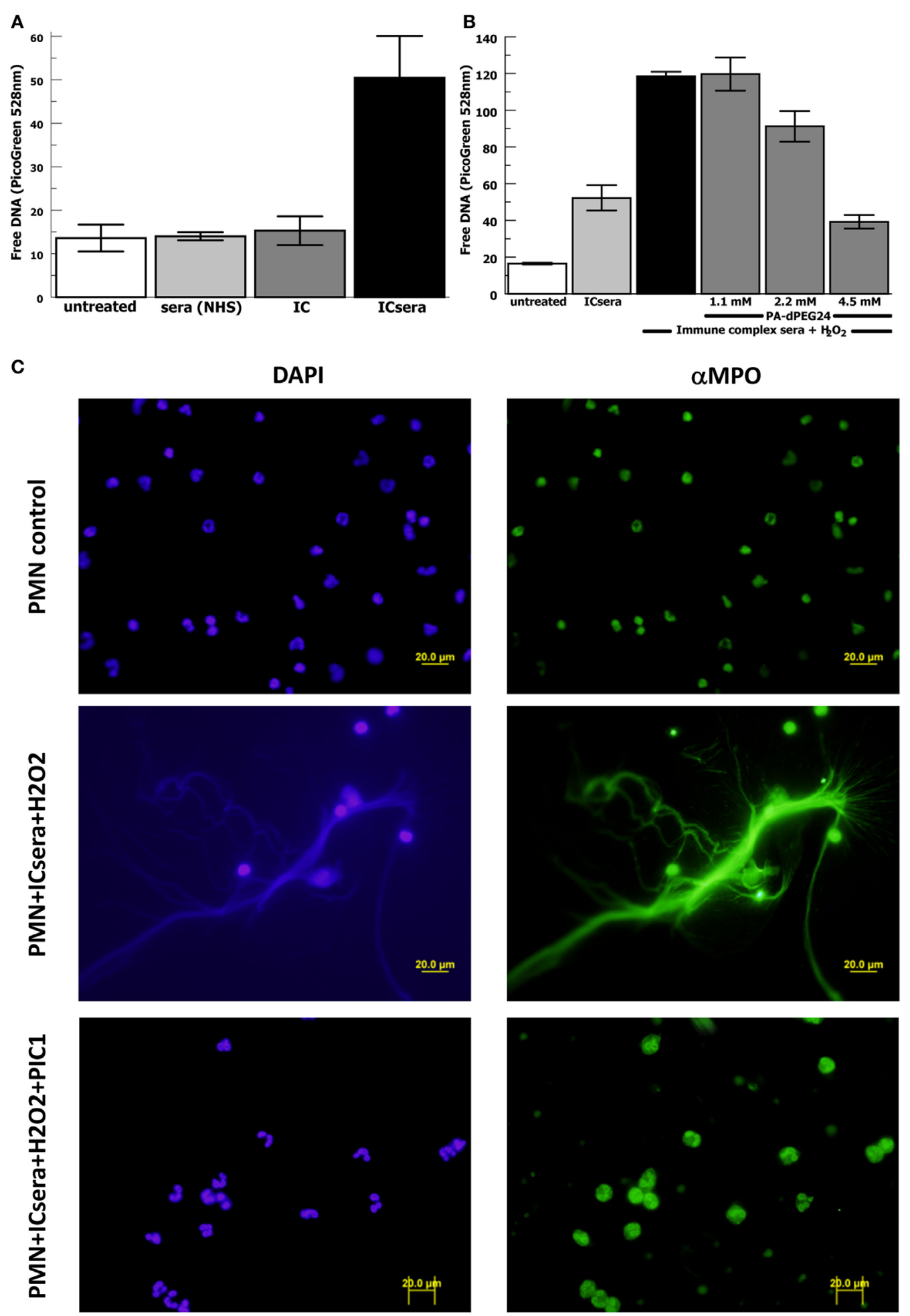

FIGURE 7 | PA-dPEG24 inhibition of immune complex-activated serum-initiated neutrophil extracellular trap (NET) formation with human neutrophils (PMN) assayed by fluorescence microscopy and PicoGreen quantitation of free DNA. Ovalbumin-antiovalbumin immune complexes (IC) were utilized. (A) NET formation induced by PMNs incubated alone (untreated), with normal human sera (NHS), IC alone (IC), or immune-complex-activated human sera (ICsera). Data are means of ( $n=5)$ independent experiments \pm SEM. (B) PA-dPEG24 inhibition of NET generation by human neutrophils stimulated with ICsera assayed by PicoGreen. Data are means of $(n=4)$ independent experiments \pm SEM. (C) The first row shows unstimulated neutrophils, the second row shows neutrophils stimulated with ICsera and hydrogen peroxide $\left(\mathrm{H}_{2} \mathrm{O}_{2}\right)$, and the third row shows neutrophils stimulated with ICsera $+\mathrm{H}_{2} \mathrm{O}_{2}$ in the presence of PA-dPEG24 (peptide inhibitor of complement C1) The first column is slides probed with DAPI to visualize DNA and the second column is slides probed with anti-MPO antibody. 


\section{ETHICS STATEMENT}

Blood from healthy donors was obtained with written informed consent under an Eastern Virginia Medical School IRB approved protocol, 02-06-EX 0216.

\section{AUTHOR CONTRIBUTIONS}

$\mathrm{PH}, \mathrm{NK}$, and $\mathrm{KC}$ provided the concepts for the study, designed the tests, and wrote the manuscript. $\mathrm{PH}$ and $\mathrm{AE}$ performed

\section{REFERENCES}

1. Ballanti E, Perricone C, Greco E, Ballanti M, Di Muzio G, Chimenti MS, et al. Complement and autoimmunity. Immunol Res (2013) 56(2-3):477-91. doi:10.1007/s12026-013-8422-y

2. Barilla-Labarca ML, Toder K, Furie R. Targeting the complement system in systemic lupus erythematosus and other diseases. Clin Immunol (2013) 148(3):313-21. doi:10.1016/j.clim.2013.02.014

3. Daha NA, Banda NK, Roos A, Beurskens FJ, Bakker JM, Daha MR, et al. Complement activation by (auto-) antibodies. Mol Immunol (2011) 48(14):1656-65. doi:10.1016/j.molimm.2011.04.024

4. Zawrotniak M, Rapala-Kozik M. Neutrophil extracellular traps (NETs) formation and implications. Acta Biochim Pol (2013) 60(3):277-84.

5. Knight JS, Kaplan MJ. Lupus neutrophils: 'NET' gain in understanding lupus pathogenesis. Curr Opin Rheumatol (2012) 24(5):441-50. doi:10.1097/ BOR.0b013e3283546703

6. Lood C, Blanco LP, Purmalek MM, Carmona-Rivera C, De Ravin SS, Smith CK, et al. Neutrophil extracellular traps enriched in oxidized mitochondrial DNA are interferogenic and contribute to lupus-like disease. Nat Med (2016) 22(2):146-53. doi:10.1038/nm.4027

7. Bassi N, Del Prete D, Ghirardello A, Gatto M, Ceol M, Zen M, et al. PTX3, anti-PTX3, and anti-C1q autoantibodies in lupus glomerulonephritis. Clin Rev Allergy Immunol (2015) 49(2):217-26. doi:10.1007/s12016-015-8476-9

8. Orbai AM, Truedsson L, Sturfelt G, Nived O, Fang H, Alarcon GS, et al. AntiC1q antibodies in systemic lupus erythematosus. Lupus (2015) 24(1):42-9. doi:10.1177/0961203314547791

9. Thanei S, Vanhecke D, Trendelenburg M. Anti-C1q autoantibodies from systemic lupus erythematosus patients activate the complement system via both the classical and lectin pathways. Clin Immunol (2015) 160(2):180-7. doi:10.1016/j.clim.2015.06.014

10. Coulthard LG, Woodruff TM. Is the complement activation product C3a a proinflammatory molecule? Re-evaluating the evidence and the Myth. J Immunol (2015) 194(8):3542-8. doi:10.4049/jimmunol.1403068

11. Tralau T, Meyer-Hoffert U, Schroder JM, Wiedow O. Human leukocyte elastase and cathepsin G are specific inhibitors of C5a-dependent neutrophil enzyme release and chemotaxis. Exp Dermatol (2004) 13(5):316-25. doi:10.1111/j.0906-6705.2004.00145.x

12. Lupia E, Del Sorbo L, Bergerone S, Emanuelli G, Camussi G, Montrucchio G. The membrane attack complex of complement contributes to plasmin-induced synthesis of platelet-activating factor by endothelial cells and neutrophils. Immunology (2003) 109(4):557-63. doi:10.1046/j.1365-2567.2003.01692.x

13. Mayadas TN, Tsokos GC, Tsuboi N. Mechanisms of immune complexmediated neutrophil recruitment and tissue injury. Circulation (2009) 120(20):2012-24. doi:10.1161/CIRCULATIONAHA.108.771170

14. Chen K, Nishi H, Travers R, Tsuboi N, Martinod K, Wagner DD, et al. Endocytosis of soluble immune complexes leads to their clearance by FcgammaRIIIB but induces neutrophil extracellular traps via FcgammaRIIA in vivo. Blood (2012) 120(22):4421-31. doi:10.1182/blood-2011-12-401133

15. Behnen M, Leschczyk C, Moller S, Batel T, Klinger M, Solbach W, et al. Immobilized immune complexes induce neutrophil extracellular trap release by human neutrophil granulocytes via FcgammaRIIIB and Mac-1.J Immunol (2014) 193(4):1954-65. doi:10.4049/jimmunol.1400478

16. Kraaij T, Tengstrom FC, Kamerling SW, Pusey CD, Scherer HU, Toes RE, et al. A novel method for high-throughput detection and quantification of the assays and data collection. $\mathrm{PH}$ and $\mathrm{KC}$ performed the analysis and statistics. All authors reviewed the manuscript for content, provided suggestions, and approved the final manuscript.

\section{FUNDING}

This work was funded in part by a grant from the Children's Hospital of The King's Daughters.

neutrophil extracellular traps reveals ROS-independent NET release with immune complexes. Autoimmun Rev (2016) 15(6):577-84. doi:10.1016/j. autrev.2016.02.018

17. Aleyd E, Al M, Tuk CW, van der Laken CJ, van Egmond M. IgA complexes in plasma and synovial fluid of patients with rheumatoid arthritis induce neutrophil extracellular traps via FcalphaRI. J Immunol (2016) 197(12):4552-9. doi:10.4049/jimmunol.1502353

18. Yuen J, Pluthero FG, Douda DN, Riedl M, Cherry A, Ulanova M, et al. NETosing neutrophils activate complement both on their own NETs and bacteria via alternative and non-alternative pathways. Front Immunol (2016) 7:137. doi:10.3389/fimmu.2016.00137

19. Akong-Moore K, Chow OA, von Kockritz-Blickwede M, Nizet V. Influences of chloride and hypochlorite on neutrophil extracellular trap formation. PLoS One (2012) 7(8):e42984. doi:10.1371/journal.pone.0042984

20. Kirchner T, Moller S, Klinger M, Solbach W, Laskay T, Behnen M. The impact of various reactive oxygen species on the formation of neutrophil extracellular traps. Mediators Inflamm (2012) 2012:849136. doi:10.1155/2012/849136

21. Parker H, Dragunow M, Hampton MB, Kettle AJ, Winterbourn CC. Requirements for NADPH oxidase and myeloperoxidase in neutrophil extracellular trap formation differ depending on the stimulus. J Leukoc Biol (2012) 92(4):841-9. doi:10.1189/jlb.1211601

22. Parker H, Winterbourn CC. Reactive oxidants and myeloperoxidase and their involvement in neutrophil extracellular traps. Front Immunol (2012) 3: 424. doi:10.3389/fimmu.2012.00424

23. Sharp JA, Whitley PH, Cunnion KM, Krishna NK. Peptide inhibitor of complement $\mathrm{cl}$, a novel suppressor of classical pathway activation: mechanistic studies and clinical potential. Front Immunol (2014) 5:406. doi:10.3389/ fimmu.2014.00406

24. Mauriello CT, Pallera HK, Sharp JA, Woltmann JL Jr, Qian S, Hair PS, et al. A novel peptide inhibitor of classical and lectin complement activation including ABO incompatibility. Mol Immunol (2013) 53(1-2):132-9. doi:10.1016/j. molimm.2012.07.012

25. Sharp JA, Hair PS, Pallera HK, Kumar PS, Mauriello CT, Nyalwidhe JO, et al. Peptide inhibitor of complement C1 (PIC1) rapidly inhibits complement activation after intravascular injection in rats. PLoS One (2015) 10(7):e0132446. doi:10.1371/journal.pone.0132446

26. Kumar PS, Pallera HK, Hair PS, Rivera MG, Shah TA, Werner AL, et al. Peptide inhibitor of complement $\mathrm{C} 1$ modulates acute intravascular hemolysis of mismatched red blood cells in rats. Transfusion (2016) 56(8):2133-45. doi:10.1111/trf.13674

27. Hair PS, Sass LA, Krishna NK, Cunnion KM. Inhibition of myeloperoxidase activity in cystic fibrosis sputum by peptide inhibitor of complement C1 (PIC1). PLoS One (2017) 12(1):e0170203. doi:10.1371/journal.pone. 0170203

28. Hair PS, Cunnion KM, Krishna NK. Peptide inhibitor of complement C1 (PIC1) inhibits the peroxidase activity of hemoglobin and myoglobin. Int J Pept (2017) 2017:9454583. doi:10.1155/2017/9454583

29. Cunnion KM, Lee JC, Frank MM. Capsule production and growth phase influence binding of complement to Staphylococcus aureus. Infect Immun (2001) 69(11):6796-803. doi:10.1128/IAI.69.11.6796-6803.2001

30. Bonaparte RS, Hair PS, Banthia D, Marshall DM, Cunnion KM, Krishna NK. Human astrovirus coat protein inhibits serum complement activation via C1, the first component of the classical pathway. J Virol (2008) 82(2):817-27. doi:10.1128/JVI.01847-07 
31. Kumar PS, Mauriello CT, Hair PS, Rister NS, Lawrence C, Raafat RH, et al. Glucose-based dialysis fluids inhibit innate defense against Staphylococcus aureus. Mol Immunol (2015) 67(2 Pt B):575-83. doi:10.1016/j. molimm.2015.07.017

32. Hair PS, Echague CG, Sholl AM, Watkins JA, Geoghegan JA, Foster TJ, et al. Clumping factor A interaction with complement factor I increases C3b cleavage on the bacterial surface of Staphylococcus aureus and decreases complement-mediated phagocytosis. Infect Immun (2010) 78(4):1717-27. doi:10.1128/IAI.01065-09

33. Carlin JB, Doyle LW. Statistics for clinicians: 4: basic concepts of statistical reasoning: hypothesis tests and the t-test. J Paediatr Child Health (2001) 37(1):72-7. doi:10.1046/j.1440-1754.2001.00634.x

34. Bergseth G, Ludviksen JK, Kirschfink M, Giclas PC, Nilsson B, Mollnes TE. An international serum standard for application in assays to detect human complement activation products. Mol Immunol (2013) 56(3):232-9. doi:10.1016/j.molimm.2013.05.221

35. Steil AA, Garcia Rodriguez MC, Alonso A, Crespo MS, Bosca L. Plateletactivating factor: the effector of protein-rich plasma extravasation and nitric oxide synthase induction in rat immune complex peritonitis. Br JPharmacol (1995) 114(4):895-901. doi:10.1111/j.1476-5381.1995. tb13288.x
36. Bestebroer J, Aerts PC, Rooijakkers SH, Pandey MK, Kohl J, van Strijp JA, et al. Functional basis for complement evasion by staphylococcal superantigen-like 7 . Cell Microbiol (2010) 12(10):1506-16. doi:10.1111/j.1462-5822.2010.01486.x

37. Brinkmann V, Zychlinsky A. Neutrophil extracellular traps: is immunity the second function of chromatin? J Cell Biol (2012) 198(5):773-83. doi:10.1083/ jcb. 201203170

38. Gupta S, Kaplan MJ. The role of neutrophils and NETosis in autoimmune and renal diseases. Nat Rev Nephrol (2016) 12(7):402-13. doi:10.1038/ nrneph.2016.71

Conflict of Interest Statement: The authors declare that the research was conducted in the absence of any commercial or financial relationships that could be construed as a potential conflict of interest.

Copyright (C) 2018 Hair, Enos, Krishna and Cunnion. This is an open-access article distributed under the terms of the Creative Commons Attribution License (CC BY). The use, distribution or reproduction in other forums is permitted, provided the original author(s) and the copyright owner are credited and that the original publication in this journal is cited, in accordance with accepted academic practice. No use, distribution or reproduction is permitted which does not comply with these terms. 